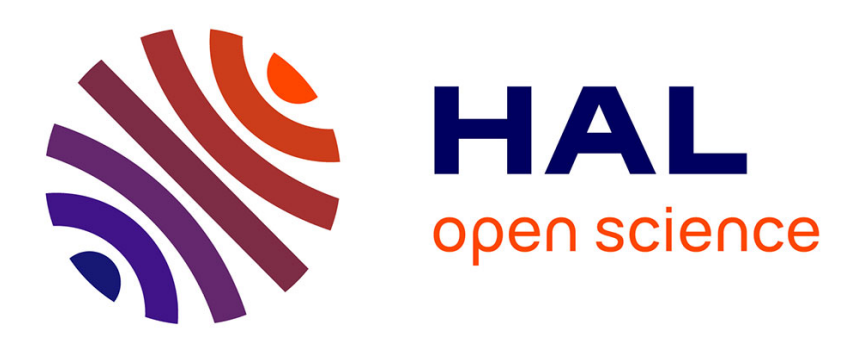

\title{
Extending standard urban outdoor noise propagation models to complex geometries
}

\author{
Matthew Kamarath, Philippe Jean, Julien Maillard, Judicaël Picaut, \\ Christophe Langrenne
}

\section{- To cite this version:}

Matthew Kamarath, Philippe Jean, Julien Maillard, Judicaël Picaut, Christophe Langrenne. Extending standard urban outdoor noise propagation models to complex geometries. Journal of the Acoustical Society of America, 2018, 143 (4), pp.2066-2075. 10.1121/1.5027826 . hal-03288502

\section{HAL Id: hal-03288502 https://hal.science/hal-03288502}

Submitted on 16 Jul 2021

HAL is a multi-disciplinary open access archive for the deposit and dissemination of scientific research documents, whether they are published or not. The documents may come from teaching and research institutions in France or abroad, or from public or private research centers.
L'archive ouverte pluridisciplinaire HAL, est destinée au dépôt et à la diffusion de documents scientifiques de niveau recherche, publiés ou non, émanant des établissements d'enseignement et de recherche français ou étrangers, des laboratoires publics ou privés. 


\title{
Extending standard urban outdoor noise propagation models to complex geometries ${ }^{\mathrm{a})}$
}

\author{
Matthew Kamrath, ${ }^{\mathrm{b}}$ Philippe Jean, and Julien Maillard \\ CSTB, 24 Rue Joseph Fourier, Saint-Martin-d'Hères 38400, \\ France \\ Judicaël Picaut \\ IFSTTAR, AME, LAE, Bouguenais 44344, France \\ Christophe Langrenne \\ LMSSC/CNAM, Paris Cedex 03 75141, France
}

(Dated: 26 February 2018) 
A hybrid method that combines a noise engineering method and the $2.5 \mathrm{D}$ boundary element method approximates outdoor sound propagation in large domains with complex objects more accurately than noise engineering methods alone and more efficiently than reference methods alone. Noise engineering methods (e.g. ISO 96132 or CNOSSOS-EU) efficiently approximate sound levels from roads, railways, and industrial sources in cities for simple, box-shaped geometries by first finding the propagation paths between the source and receiver, then applying attenuations (e.g. geometrical divergence and atmospheric absorption) to each path, and finally incoherently summing all of the path contributions. Standard engineering methods cannot model more complicated geometries, but introducing an additional attenuation term quantifies the influence of complex objects. Calculating this extra attenuation term requires reference calculations, but performing reference computations for each path is too computationally expensive. Thus, the extra attenuation term is linearly interpolated from a data table containing the corrections for many source/receiver positions and frequencies. The 2.5D boundary element method produces the levels for the real and simplified geometries, and subtracting them yields a table of corrections. For a T-shaped barrier with two buildings, this approach reduces the mean error by approximately $2 \mathrm{dBA}$ compared to a standard engineering method.

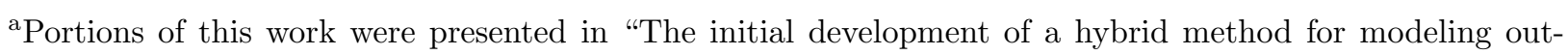
door sound propagation in urban areas," 170th Meeting of the Acoustical Society of America, Jacksonville, Florida, November 2015; "Modeling outdoor sound propagation in urban environments," 171st Meeting of the Acoustical Society of America, Salt Lake City, Utah, May 2016; "Augmenting Road Noise Engineering Methods using the Boundary Element Method," Inter-noise, Hamburg, Germany, August 2016; and "En2 abling noise engineering methods to model complex geometries," 3rd Joint Meeting of the Acoustical Society 


\section{INTRODUCTION}

Excessive environmental noise exposure from road, rail, airport, and industrial sources is a major health concern. In Western Europe, the World Health Organization estimates that over 1 million healthy life years are lost annually due to increased annoyance, sleep disturbance, and other negative health outcomes from traffic-related noise ${ }^{1}$. In response, the European Parliament passed the Environmental Noise Directive 2002/49/EC ${ }^{2}$ to evaluate and mitigate current noise levels and developed the Common Noise Assessment Methods in Europe $(\text { CNOSSOS-EU) })^{3}$ to standardize noise prediction models.

However, modeling urban outdoor sound propagation is very difficult because cities are large compared to a wavelength and contain complicated geometries. Specifically, the domain is three dimensional and is the size of multiple city blocks (i.e. at least $100 \mathrm{~m}$ ), and the frequency range goes up to the $4 \mathrm{kHz}$ octave-band, so in air the wavelength can be less than $0.1 \mathrm{~m}$. Thus, common reference approaches in outdoor sound propagation that discretize the entire domain with multiple points per wavelength like the finite element ${ }^{4,5}$, boundary element $^{6,7}$, and finite-difference methods ${ }^{8,9}$ are prohibitively expensive.

In addition, urban scenes often have complicated geometries and impedances, which must be modeled accurately because they can significantly impact the level at the receiver ${ }^{10}$. A principal example is a complex noise barrier, which could be a T-shaped barrier ${ }^{11}$ or even a barrier with an optimized shape $\mathrm{e}^{12,13}$. This criterion eliminates the engineering methods (e.g. CNOSSOS-EU, ISO 9613-2 ${ }^{14}$, and Harmonoise ${ }^{15,16}$ ) because they are not designed to model such complex shapes and geometrical approaches more generally (e.g. ray tracing $^{17,18}$ or 
beam tracing ${ }^{19,20}$ ) because these complex shapes would require too many diffracting edges to model them efficiently. In addition, the parabolic equation method ${ }^{21,22}$ cannot model such complex geometries because it usually only models one-way propagation ${ }^{23}$ to improve computational efficiency.

In addition to these standard approaches, many hybrid methods cannot model large 3D domains with complex geometries. Many hybrid approaches combine methods using spatial decomposition, which applies a reference method to geometrically complex regions and an efficient method to geometrically simple regions. For example, using spatial decomposition one can combine the finite-difference time-domain and parabolic equation methods ${ }^{24}$, the boundary element and parabolic equation methods ${ }^{25}$, or the boundary element and ray tracing methods ${ }^{26-28}$. All of these methods use a reference method to calculate the pressure on a fictitious surface around the complex area and propagate those pressures using an efficient method. However, the number of points on this fictitious surface increases with frequency, so for high frequencies and 3D geometries the cost can be prohibitive. Indeed, most of the applications in those papers are only two dimensional or only use low frequencies.

To mitigate this problem, Yeh et al..$^{29}$ apply both spatial and frequency decomposition, which uses a reference method for low frequencies near complex objects and an efficient method otherwise. This approach makes the cost scale linearly with the largest dimension of the domain instead of the domain volume, but it still requires expensive reference method calculation for every new scene. Performing a reference calculations for each complex object instead of each scene is potentially much less expensive but also less accurate. 
The literature proposes two principle ways to quantify the influence of a complex object. The first method performs a large number of reference calculations and then fits an analytical curve to the data ${ }^{30,31}$. Evaluating the function at a given source and receiver yields the associated correction. This approach has the potential to substantially reduce the amount of data that must be stored because the data is approximated with an analytical curve. However, this approach limits the number of data points to about 10,000 because it requires a matrix inversion, which uses $O\left(n^{3}\right)$ operations. The second method stores a large table of reference computations and then interpolates the results depending on the source/receiver positions and frequency ${ }^{32,33}$. In this approach, the number of data points is only limited by the available computer memory. Thus, the hybrid method tabulates many reference results and interpolates them.

The current literature is limited because it does not explain how to apply the calculated corrections in a complex urban scene with other objects and does not test the approach. This article seeks to fill this gap by developing and validating a hybrid approach that combines an engineering method and the 2.5D boundary element method. To that end, Section II explicates the hybrid method and Section III evaluates it for three test cases using a Tbarrier.

\section{THE HYBRID METHOD}

To determine the level at a receiver, engineering methods 1) input the scene information, 2) find the propagation paths between the source and receiver, 3) calculate the attenuations for each path, and 4) sum the contributions of each propagation path. Summing the 
directional source level and attenuations yields the path contributions:

$$
L=L_{\mathrm{w}}+A_{\mathrm{div}}+A_{\mathrm{atm}}+A_{\text {boundary }},
$$

where $L$ is the sound pressure level at the receiver, $L_{\mathrm{w}}$ is the directional sound pressure level of the source, $A_{\text {div }}$ is the attenuation due to geometrical divergence, $A_{\text {atm }}$ is the attenuation due to atmospheric absorption, and $A_{\text {boundary }}$ is the attenuation due to the ground effect, reflecting from vertical surfaces, and diffracting over/around objects. All of the terms are in decibels and typically computed for each octave or third-octave band.

The hybrid method described here combines an engineering method and a reference method to efficiently approximate outdoor noise propagation with complex objects. The hybrid method modifies Equation (1) by introducing an extra attenuation term for the complex objects:

$$
L=L_{\mathrm{w}}+A_{\mathrm{div}}+A_{\mathrm{atm}}+A_{\text {boundary }}+A_{\text {extra }},
$$

where $A_{\text {extra }}$ is the attenuation due to complex object compared to a simplified object. Calculating this term requires reference computations that can model the complex shape accurately, but performing a reference calculation for every propagation path would be prohibitively expensive. Instead, the hybrid method tabulates a large number of reference results, which is then interpolated as needed.

Thus, the hybrid method requires some reference computations before the engineering method begins. First, the reference method [e.g. the boundary element method (BEM)] calculates the attenuation of the complex object compared to a simple object for a large number of source/receiver locations and frequencies. Second, the data is sorted and converted to frequency-band data for the engineering method. These expensive steps are only 
required once for each complex object because the results can be stored for later use. Then, the engineering method can proceed. Figure 1 outlines these steps.

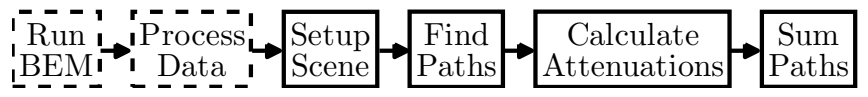

FIG. 1. Outline of the hybrid method. The dashed boxes indicate that these steps are not always required.

After the engineering method finds all the paths, the extra attenuation must be calculated for each path just like all of the other attenuations. If a propagation path diffracts over a complex object that has the largest path length difference of any object in that path, then the extra attenuation could be non-zero; otherwise, the extra attenuation is zero. If the extra attenuation could be non-zero, then the engineering method uses the path geometry to find the local source/receiver position. Then, linearly interpolating the table of corrections at that position produces the extra attenuation.

The rest of this section gives greater detail about the hybrid method. Specifically, Section II A explains how to calculate the table of corrections, Section II B elucidates how to convert the source/receiver coordinates in the engineering method to the coordinates in the table of corrections, Section II C describes how to interpolate the table of corrections to produce the extra attenuation, and Section IID discusses what to do when a point is outside of the table of corrections. 
Outdoor noise propagation with complex geometries

\section{A. Calculating the table of corrections}

The first step in the hybrid method is calculating the table of corrections. While in general any approach capable of modeling complex geometries and arbitrary impedances could be used, the 2.5D boundary element method (BEM) is particularly well suited for this application. First, BEM efficiently models infinite domains, which are required in outdoor sound propagation, by imposing the Sommerfeld radiation conditions to only allow outgoing waves and by modifying the free space Green's function using an image source to model infinite flat ground. Second, 2.5D BEM ${ }^{10}$ models infinitely long objects with an arbitrary, constant cross-section as an integral of $2 \mathrm{D}$ problems ${ }^{34}$. Thus, instead of using $3 \mathrm{D}$ elements throughout the domain, 2.5D BEM only has to mesh the $1 \mathrm{D}$ perimeter of the object's crosssection. For computational efficiency, the complex geometries are restricted to objects that are long compared to a wavelength and have a constant (arbitrary) cross-section along the long dimension. Using 2.5D BEM also allows the source to be a point source instead of a coherent line source, which affects the predicted attenuation ${ }^{35}$.

The next step is to define the variables for the table of corrections. The source/receiver positions and the frequency clearly affect the attenuation of a complex object, which in arbitrary 3D space requires seven variables. However, since the object is infinitely long in one direction, only the relative position of the source and receiver is required parallel to the object. Thus, there are six variables (Figure 2): $x_{\mathrm{s}}$ and $x_{\mathrm{r}}$ are the perpendicular displacements (i.e. $x_{\mathrm{s}}$ is negative and $x_{\mathrm{r}}$ is positive) of the source and receiver from the object, $z_{\mathrm{s}}$ and $z_{\mathrm{r}}$ are the heights of the source and receiver relative to the bottom of the 
complex object, $\Delta y$ is the distance between the source and receiver that is parallel to the complex object, and $f$ is the frequency.

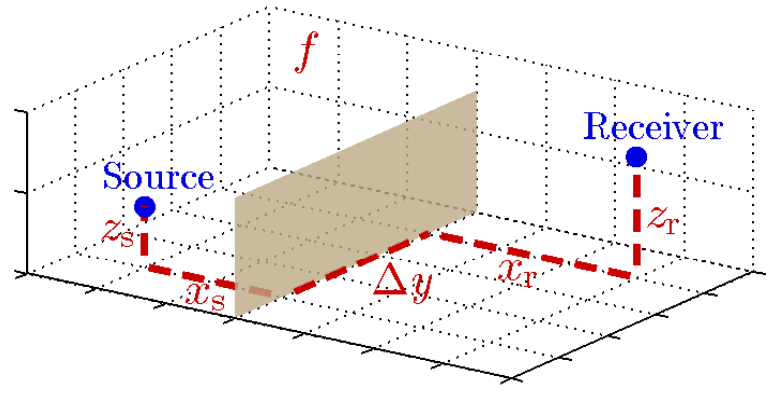

FIG. 2. (Color online) The variable definitions for the table of corrections.

The next step is to define the source and receiver positions. To minimize the memory and time costs of interpolating the data, the data points are constrained to a non-uniform grid. The range of each variable depends on the application; for example, the source/receiver positions may be different for modeling road noise versus industrial noise. Also, since the extra attenuation probably changes most rapidly near the complex object and the ground, the values of the individual variables follow an exponential function for simplicity, which Section III demonstrates. With six variables, the amount of data increases very rapidly with the number of points per variable. Using only 20 points per variable, the total number of points would be $20^{6}=6.4 \times 10^{7}$ points.

The last step before calculating the points is deciding the geometry of the simplified object and the surroundings. The simplified object changes depending on the complex object, but 1) the engineering method must be able to model it and 2) it should be as similar as possible to the complex object. For example, the simplified object for a complex barrier would be a straight barrier (i.e. an I-barrier) that is the same height as the complex 
barrier. The complex and simplified objects should be similar because the hybrid method assumes that the extra attenuation is mostly independent of the surrounding geometry. This assumption is not true in general but is approximately true for sufficiently similar geometries. For simplicity and computational efficiency, if the ground is approximately flat, then the surrounding geometry is hard flat ground. Section III B suggests that hard, flat ground even gives reasonable results when the eventual complex surroundings do not have hard ground. The most important reason for only using hard ground is that it drastically decreases the number of necessary tables of corrections. If the ground is not flat, then the non-flat portions would need to be meshed in BEM, which is possible but more costly.

After performing the BEM computations, the data must be converted from single frequency calculations to octaves or third-octaves to interface with the engineering methods. Given $N$ complex pressures $p_{i}$ that are computed at the linearly spaced frequencies $f_{i}$ in the frequency range $\left[f_{\min }, f_{\max }\right]$ (i.e. $f_{i}=\frac{f_{\max }-f_{\min }}{N}\left(i-\frac{1}{2}\right)$ for $\left.i=[1, N]\right)$ and the reference pressure $p_{\text {ref }}$, the corresponding frequency band level is

$$
L=10 \log _{10}\left(\frac{f_{\mathrm{max}}-f_{\mathrm{min}}}{N} \sum_{i=1}^{N} \frac{\left|p_{i}\right|^{2}}{p_{\mathrm{ref}}^{2}}\right),
$$

where $N$ is sufficiently large for the sum to converge, which is typically about ten frequencies. This calculation is performed for each frequency band, for each source/receiver pair, and for both the simplified and complex cases. Then, subtracting the levels for the simplified case $L_{\text {simplified }}$ from the levels for the complex case $L_{\text {complex }}$ for every frequency and source/receiver pair yields the table of corrections $\Delta L$ :

$$
\Delta L=L_{\text {complex }}-L_{\text {simplified }}
$$


Here, $\Delta L$ refers to values that depend on the source/receiver positions and frequency and that are stored in a table, which is later interpolated to determine the extra attenuation $A_{\text {extra }}$ of a given propagation path. The table of corrections must be interpolated because the propagation path could have a source/receiver location that is not in the table.

Finally, the points are sorted based on the source/receiver locations to make interpolating the data more efficient.

\section{B. Local source/receiver positions}

The variables in the table of corrections (i.e. $x_{\mathrm{s}}, z_{\mathrm{s}}, x_{\mathrm{r}}, z_{\mathrm{r}}, \Delta y$ ) are defined relative to the complex object, but the variables that the engineering method uses are not. Specifically, the engineering method uses two sets of coordinates (Figure 3). First, it uses standard Cartesian coordinates $\left(x_{\text {global }}, y_{\text {global }}, z_{\text {global }}\right)$ for all of the objects where the origin and orientation of the axes are arbitrarily chosen by the user. In addition, all of the points along a propagation path also get a second pair of coordinates $\left(d_{\text {path }}, z_{\text {path }}\right)$ where $d_{\text {path }}$ is the distance along the path and $z_{\text {path }}$ is the height of the path. Thus, the global variables of the engineering method must be converted to the local coordinates of the table of corrections to interpolate it.

The first step is to determine the smallest angle $\theta$ between the complex object and the propagation path (Figure 3). Using the dot product and the locations of the ends of the complex object, the source, and the receiver, the angle $\theta$ is

$$
\theta=\cos ^{-1}\left|\hat{\mathbf{r}}_{\text {global }, s \rightarrow r} \cdot \hat{\mathbf{r}}_{\text {global }, b_{1} \rightarrow b_{2}}\right|
$$


where $\hat{\mathbf{r}}_{\text {global }, r \rightarrow s}$ is a $2 \mathrm{D}\left(x_{\text {global }}, y_{\text {global }}\right)$ unit vector parallel to the propagation path at the diffraction point and $\hat{\mathbf{r}}_{\text {global }, b_{1} \rightarrow b_{2}}$ is a $2 \mathrm{D}$ unit vector parallel to the complex object at the diffraction point. If there are more than three propagation points $\left[\right.$ e.g. $\left(s, p_{1}, d, p_{2}, r\right)$ instead of $(s, d, r)$ where $p_{1}$ is a reflection point and $p_{2}$ is a lateral diffraction point], then $\hat{\mathbf{r}}_{\text {global }, s \rightarrow r}$ would become $\hat{\mathbf{r}}_{\text {global }, p_{1} \rightarrow p_{2}}$ to keep the unit vector parallel to the propagation path at the diffraction point.

After calculating $\theta$, applying geometry in Figure 3 yields all the local variables:

$$
\begin{aligned}
& x_{s}=\left[d_{\text {path }}(s)-d_{\text {path }}(d)\right] \sin \theta, \\
& z_{s}=z_{\text {path }}(s), \\
& x_{r}=\left[d_{\text {path }}(r)-d_{\text {path }}(d)\right] \sin \theta, \\
& z_{r}=z_{\text {path }}(r), \\
& \Delta y=\left[d_{\text {path }}(r)-d_{\text {path }}(s)\right] \cos \theta,
\end{aligned}
$$

where $d_{\text {path }}(s), d_{\text {path }}(d)$, and $d_{\text {path }}(r)$ are $d_{\text {path }}$ at the source, diffraction point, and receiver; and $z_{\text {path }}(s)$ and $z_{\text {path }}(r)$ are $z_{\text {path }}$ at the source and receiver. Since $d_{\text {path }}$ increases from the source to the receiver, all of the variables are positive except $x_{\mathrm{s}}$, which is always negative.

\section{Linear interpolation}

Once the local source/receiver position $\left(\mathbf{x}=x_{\mathrm{s}}, z_{\mathrm{s}}, x_{\mathrm{r}}, z_{\mathrm{r}}, \Delta y\right)$ is determined, the next step is to interpolate the table of corrections at this point. The hybrid method uses $k$-linear spline interpolation where $k$ is the number of dimensions because this interpolation method 

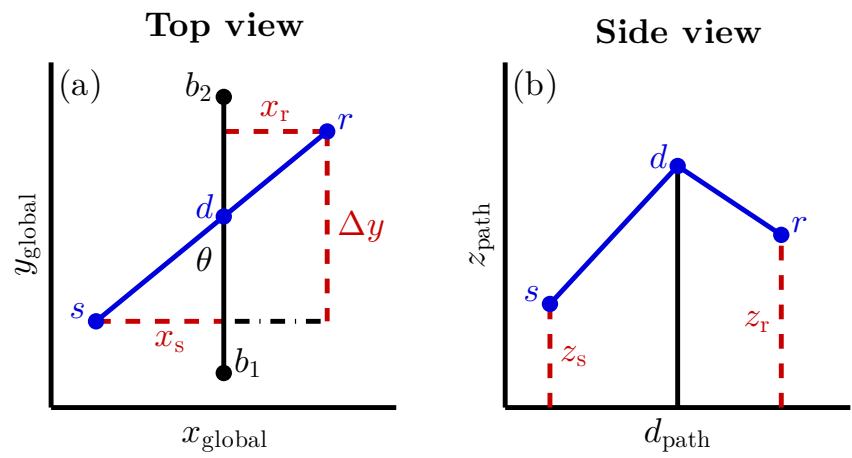

FIG. 3. (Color online) Converting the global coordinates used in the engineering method to the local coordinates used in the table of corrections.

works well for data on a grid. Although the real application has five dimensions (i.e. $k=5$ ), first consider a simpler 2D example.

Spline interpolation breaks the entire region into smaller subregions and only uses the nearby data points. For 2D data on a grid, each of the subregions is a rectangle with a data point at each corner. Each data point $\left(x_{1}, x_{2}\right)$ is uniquely identified using two indices $\left(i_{1}, i_{2}\right)$ just like in a $2 \mathrm{D}$ matrix. The corners of a rectangle are the data points with the indices $\left(i_{1}, i_{2}\right),\left(i_{1}, i_{2}+1\right),\left(i_{1}+1, i_{2}\right)$, and $\left(i_{1}+1, i_{2}+1\right)$, so each rectangle can be uniquely identified using the smallest indices $\left(i_{1}, i_{2}\right)$ of the rectangle. For each dimension, the smallest index of a rectangle is the largest index that has a position less than or equal to the position of the interpolation point.

Given an interpolation point, the first step is to determine the indices of the rectangle that contains the interpolation point. For an arbitrary grid, the most efficient method is to use a binary search for each dimension, which uses $O(\log n)$ operations. Assuming sorted data, a binary search starts at the center index and determines if the position of that index 
is too small, the correct point, or too big. If the position is the correct point, then the search algorithm terminates. Otherwise, the process repeats with the remaining data (i.e. the data that is larger if the position is too small or the data that is smaller if the position is too large), which is cut in half at each iteration.

After identifying the rectangle that contains the interpolation point, the next step is to use the data at the corners of the rectangle to approximate the value at the interpolation point. In $2 \mathrm{D}, k$-linear interpolation method is called bilinear interpolation, and the interpolated value is

$$
y\left(x_{1}, x_{2}\right)=c_{0,0}+c_{1,0} x_{1}+c_{0,1} x_{2}+c_{1,1} x_{1} x_{2},
$$

where the $c$ 's are unknown coefficients and the $x$ 's are the inputs. In the real application, $y$ is $A_{\text {extra }}$ or $\Delta L$ and the $x$ 's are $x_{\mathrm{s}}, z_{\mathrm{s}}, x_{\mathrm{r}}, z_{\mathrm{r}}$, or $\Delta y$. Evaluating Equation (7) at the four corners of the rectangle that contains the interpolation point yields a system of four equations with four unknown coefficients. Rescaling the $x$ 's to be between zero and one simplifies the matrix equation to

$$
\left[\begin{array}{l}
c_{0,0} \\
c_{0,1} \\
c_{1,0} \\
c_{1,1}
\end{array}\right]=\left[\begin{array}{rrrr}
1 & 0 & 0 & 0 \\
-1 & 1 & 0 & 0 \\
-1 & 0 & 1 & 0 \\
1 & -1 & -1 & 1
\end{array}\right]\left[\begin{array}{l}
y(0,0) \\
y(0,1) \\
y(1,0) \\
y(1,1)
\end{array}\right] .
$$

Plugging the values of the coefficients from Equation (8) into Equation (7) yields the function to interpolate the value at any location inside the rectangle. 
Applying the same procedure to the general dimensional case allows this interpolation method to model the 5D application. First, determine the indices $\left(i_{1}, i_{2}, i_{3}, i_{4}, i_{5}\right)$ of the hyper-rectangle using a binary search for each dimension. Instead of just $2^{2}=4$ points, this hyper-rectangle contains the $2^{5}=32$ points [i.e. $\left(i_{1}, i_{2}, i_{3}, i_{4}, i_{5}\right),\left(i_{1}, i_{2}, i_{3}, i_{4}, i_{5}+1\right), \ldots\left(i_{1}+\right.$ $\left.\left.1, i_{2}+1, i_{3}+1, i_{4}+1, i_{5}+1\right)\right]$. Second, interpolate using the data at the corners of the hyper-rectangle. The general dimensional form of Equation (7) is

$$
y(\mathbf{x})=\sum_{m_{1}, m_{2}, \ldots m_{k}=0}^{1} c_{m_{1}, m_{2}, \ldots m_{k}} x_{1}^{m_{1}} x_{2}^{m_{2}} \ldots x_{k}^{m_{k}}
$$

where the sum is over all $2^{k}$ combinations of the $m$ 's being zero or one. Evaluating this equation at the $2^{k}$ corners of the hyper-rectangle that contains the interpolation point yields the $2^{k}$ coefficients $c_{m_{1}, m_{2}, \ldots m_{k}}$. This matrix equation can be solved analytically for an arbitrary number of dimensions when all of the coordinates $\mathbf{x}$ are rescaled to be between zero and one. Specifically,

$$
\left[\begin{array}{c}
c_{0,0, \ldots 0} \\
c_{0,0, \ldots 1} \\
\vdots \\
c_{1,1, \ldots 1}
\end{array}\right]=\mathbf{A}_{k}\left[\begin{array}{c}
y(0,0, \ldots 0) \\
y(0,0, \ldots 1) \\
\vdots \\
y(1,1, \ldots 1)
\end{array}\right],
$$

where $\mathbf{A}_{k}$ is found using the following recursive relationship:

$$
\mathbf{A}_{0}=1 \text { and } \mathbf{A}_{n+1}=\left[\begin{array}{cc}
\mathbf{A}_{n} & 0 \\
-\mathbf{A}_{n} & \mathbf{A}_{n}
\end{array}\right] \text {. }
$$


For the current application, the $y$ 's in Equation (10) are the $\Delta L$ 's from the table of corrections.

Finally, plugging the coefficients from Equation (10) into Equation (9) and evaluating it at the rescaled interpolation point yields the interpolated value, which is the extra attenuation $A_{\text {extra }}$ for that propagation path.

\section{Extrapolation}

Sometimes, the desired source/receiver pair falls outside of the range of the table of corrections. When this occurs, linear interpolation may produce large errors, especially when the point is far away from the points in the dataset. If many points are outside of the range of the table of corrections, then the range should be expanded to include these points. If some points are still outside of the range of the table of corrections, then simplest solution is to set the extra attenuation to zero outside of the table of corrections. This approach is discontinuous at the boundary of the dataset, so the hybrid method smooths this transition by finding the closest data point and multiplying it by a decay function:

$$
f(d)=\frac{1}{1+a d},
$$

where $d$ is the standard Euclidean distance extended to 5D between the desired point and the closest data point and $a$ is a positive coefficient, which can be set to $1 \mathrm{~m}^{-1}$. This decay function guarantees that the extrapolated values close to the data are similar to the value of the closest data point but extrapolated values far from the data are close to zero. 
The exact value of $a$ is unimportant because these long propagation paths are rarely the dominate contribution at the receiver. If the value of $a$ does significantly impact the results, then the range of the variables should be increased. However, the value of $a$ can be better approximated by considering how quickly the correlation between nearby points in the dataset deteriorates as a function of distance.

\section{VALIDATION}

The first step to validate the hybrid method is to choose a complex object and to calculate its associated table of corrections. Since a T-shaped barrier has been widely studied $^{6,10,11,30,31}$, the complex object is a T-barrier that is $3 \mathrm{~m}$ tall and $0.2 \mathrm{~m}$ thick with a $1 \mathrm{~m}$ wide top, and the simplified object is an I-barrier that is $3 \mathrm{~m}$ tall and $0.2 \mathrm{~m}$ thick (Figure 4). Since the barriers are modeled in 2.5D, they have a constant cross-section and infinite length. The surround scene is flat, hard ground, and the medium is air where the sound speed is $c=340 \mathrm{~m} / \mathrm{s}$, the density is $\rho=1.3 \mathrm{~kg} / \mathrm{m}^{3}$, and the reference pressure is $p_{\text {ref }}=20 \mu \mathrm{Pa}$. The frequencies are the $50 \mathrm{~Hz}-5 \mathrm{kHz}$ third-octave bands.

The source position is $x_{\mathrm{s}}=-3.0 \mathrm{~m}$ and $z_{\mathrm{s}}=0.3 \mathrm{~m}$, and the receiver height is $z_{\mathrm{r}}=1.5 \mathrm{~m}$. Each of these variables only need one value because all of the following examples only one source position and one receiver height. In practice, the source/receiver positions are unknown, so to compensate the total number of locations for $x_{\mathrm{r}}$ and $\Delta y$ is limited to only $20^{2}$, which would be similar to using $20^{5}$ locations if all five of the position variables were unknown. Each location also has about twenty frequencies, which would bring the total to the $20^{6}$ points that is mentioned in Section II A. Since $x_{\mathrm{r}}$ and $\Delta y$ require many values, for 

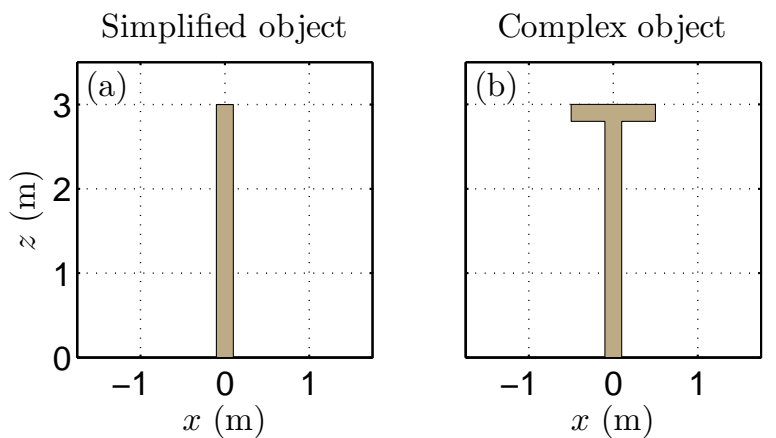

FIG. 4. (Color online) The cross-sections of (a) the simplified I-barrier and (b) the complex Tbarrier.

simplicity the following exponential equation gives their values:

$$
x_{n}=e^{\ln \left(x_{N-1}-x_{0}+1\right) \frac{n}{N-1}}+x_{0}-1,
$$

where $N$ is the number of points, $n$ is the index such that $0 \leq n \leq N-1$, and $x_{0}$ and $x_{N-1}$ are the smallest and large values along a dimension. The range of $x_{\mathrm{r}}$ is $1 \mathrm{~m}$ to $200 \mathrm{~m}$ and of $\Delta y$ is $0 \mathrm{~m}$ to $60 \mathrm{~m}$. Making the number of points along each dimension proportional to its range and keeping the total number of points less than 400 yields 36 points for $x_{\mathrm{r}}$ and 11 points for $\Delta y$. Thus, evaluating Equation (13) at $\left(x_{0}, x_{N-1}, N\right)=(1,200,36)$ for $x_{\mathrm{r}}$ and at $\left(x_{0}, x_{N-1}, N\right)=(0,60,11)$ for $\Delta y$ gives the receiver locations.

\section{A. Hard ground test case}

The first test case is a T-barrier with hard ground. It is designed to measure two sources of error: 1) the error between the engineering method and the 2.5D boundary element method for an I-barrier and 2) the interpolation error from interpolating the table of corrections. 
Outdoor noise propagation with complex geometries

Thus, the scene should be identical to the scene that is used to calculate the table of corrections.

The first test case is the infinite I-barrier or T-barrier in Figure 4 along the $y$-axis with hard ground at $z=0 \mathrm{~m}$. The source is a monopole at $(x, y, z)=(-3,17.5,0.3) \mathrm{m}$, and the receivers are on a uniform grid at $x=[0.00,0.25, \ldots 25.00] \mathrm{m}, y=[0.00,0.25, \ldots 35.00] \mathrm{m}$, and $z=1.5 \mathrm{~m}$. The frequencies are the $50 \mathrm{~Hz}-5 \mathrm{kHz}$ third-octave bands. To combine the frequencies, they are A-weighted and weighted using the combined spectrum of the engine and rolling noise of a car traveling at $80 \mathrm{~km} / \mathrm{hr}$, which the Hosanna project (see Tables 9 and 10 in Task 2.3$)^{36}$ provides. The medium is air, and the engineering method is Harmonoise with up to 6 reflections, 2 lateral diffractions, and a $1 \mathrm{~km}$ path length. However, lateral diffraction around the barrier is turned off. In addition to the hybrid method, the 2.5D boundary element method (BEM) also produces comparison reference results. The 2.5D boundary element method calculates both the table of corrections, which has 396 source/receiver pairs, and the reference results, which has 14,241 source/receiver pairs.

Figure 5 illustrates the results. The $2.5 \mathrm{D}$ boundary element method produces the top plots and the hybrid method produces the bottom plots. The left-hand plots are for the I-barrier and the right-hand plots are for the T-barrier. The hybrid method for the I-barrier is just the engineering method because the extra attenuation is always zero.

Contrasting the I-barrier plots demonstrates two main differences between 2.5D BEM and the engineering method. First, at $x=20 \mathrm{~m}$ plot (a) shows a region of increased levels that is not represented in plot (c). Considering the third-octave results, which are not shown, indicates that BEM is predicting a strong ground effect at $1.6 \mathrm{kHz}$ that the approximation 


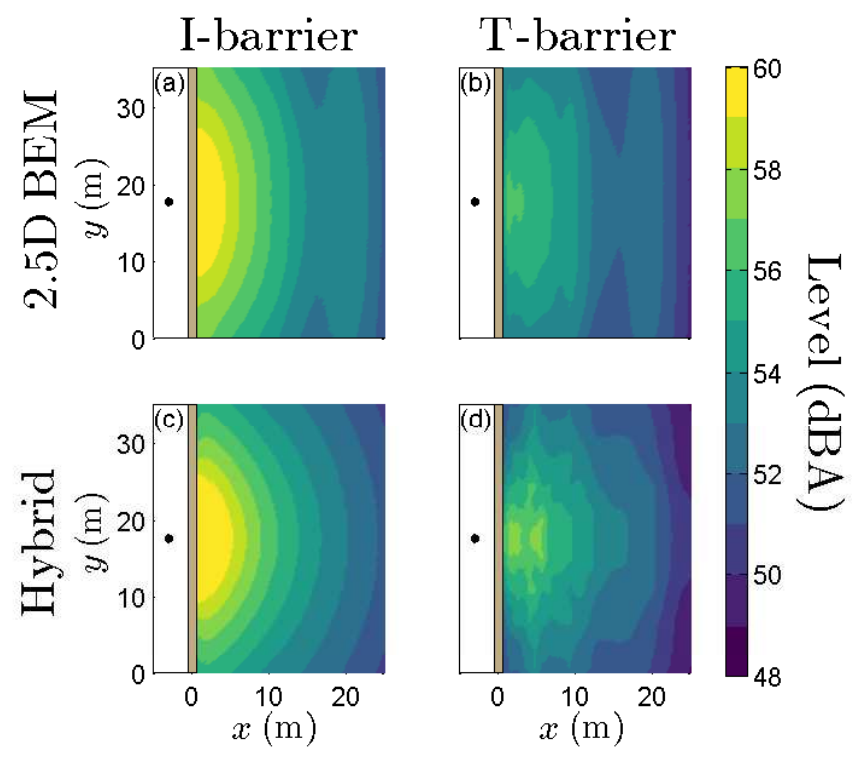

FIG. 5. (Color online) I-barrier (left) or T-barrier (right) with hard ground using 2.5D BEM (top) or the hybrid method (bottom).

in the engineering method does not capture. Second, the source appears to be spread in the $y$-direction in plot (a) but not in plot (c). This spreading occurs because of the diffraction over the top edge of the barrier. However, again the engineering method does not predict this spreading and is more circular because, for simplicity, it assumes that the barrier is perpendicular to the propagation path regardless of the incidence angle. Contrasting the T-barrier plots shows similar differences, which carry over from the I-barrier case.

The principal result in Figure 5 is that the hybrid method [plot $(d)$ ] does better than the engineering method [plot (c)] at predicting the levels of the T-barrier [plot (b)]. To make this statement quantitative, the error is defined to be

$$
E=L_{\text {model }}-L_{\text {reference }}
$$


where $L_{\text {model }}$ is the modeled level and $L_{\text {reference }}$ is the reference level. The modeled level comes from the engineering method [plot (c)] or the hybrid method [plot (d)], and the reference level comes from 2.5D BEM for the I-barrier [plot (a)] or the T-barrier [plot (b)]. Table I gives the mean $\mu$, standard deviation $\sigma$, and root mean squared (RMS) value of the error using the receivers in the range $2 \mathrm{~m} \leq x \leq 25 \mathrm{~m}$.

TABLE I. Error for the hard ground case. Here, $\mu$ is the mean error, $\sigma$ is the standard deviation, and RMS is the root mean squared error. All of the values are in dBA.

\begin{tabular}{lcccc}
\hline \hline Method & Barrier & $\mu$ & $\sigma$ & RMS \\
\hline Harmonoise & $\mathrm{I}$ & -0.28 & 0.55 & 0.62 \\
Harmonoise & $\mathrm{T}$ & 1.65 & 0.97 & 1.92 \\
Hybrid & $\mathrm{T}$ & -0.10 & 0.68 & 0.69 \\
\hline \hline
\end{tabular}

In Table I, the RMS error for the I-barrier using Harmonoise is $0.62 \mathrm{dBA}$, but it triples (1.92 dBA) for the T-barrier because the T-top is neglected in the model. This increased error is the impetus for developing the hybrid method, which reduces the RMS error to approximately the level for the I-barrier $(0.69 \mathrm{dBA})$. Moreover, the hybrid method reduces the standard deviation for the T-barrier from $0.97 \mathrm{dBA}$ to $0.68 \mathrm{dBA}$. Without interpolation error, the mean and standard deviation for the I-barrier using the engineering method would be the same as for the T-barrier using the hybrid method because the hybrid method does not remove the errors from the engineering method for the I-barrier. Since they are not equal, there is a moderate amount of interpolation error, which is smaller than the error between 
the engineering method and 2.5D BEM for the I-barrier. This interpolation error could be reduced by increasing the number of points in the table of corrections or by optimizing the locations of the points.

\section{B. Soft ground test case}

The next case is a T-barrier with soft ground and is designed to test if a table of corrections that is calculated with hard ground can be used to accurately model soft ground. Specifically, this case is identical to the previous case except that the entire ground is soft like grass with a flow resistance of $200 \mathrm{kPa} \cdot \mathrm{s} / \mathrm{m}^{2}$. 2.5D BEM approximates the ground impedance using the Delany-Bazley model ${ }^{37}$.
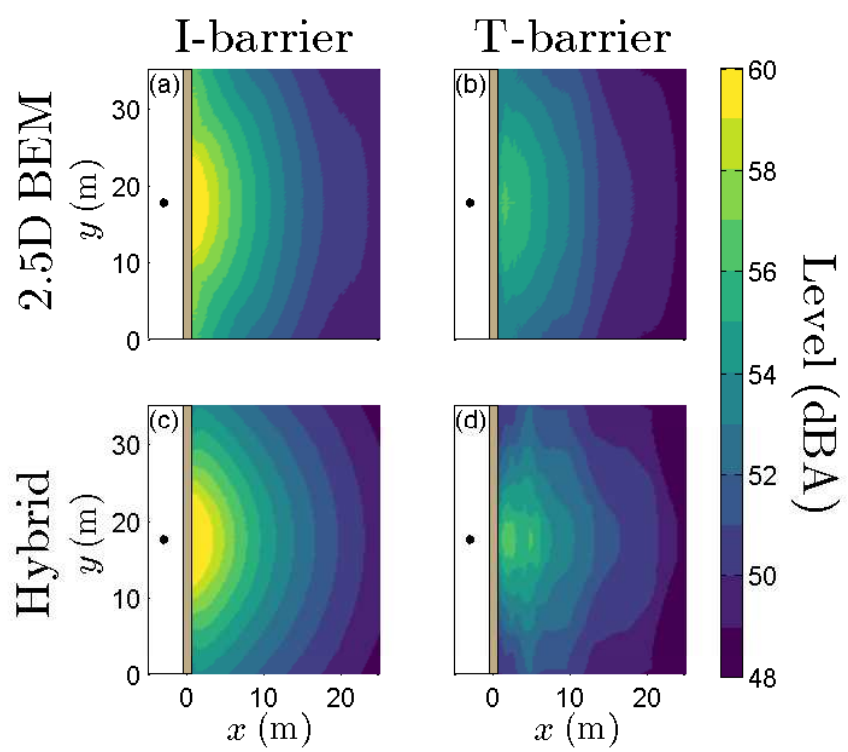

FIG. 6. (Color online) I-barrier (left) or T-barrier (right) with soft ground using 2.5D BEM (top) or the hybrid method (bottom). 
The results for the soft ground case (Figure 6) are very similar to the results for the hard ground case (Figure 5). The biggest difference is that the global level is 1-3 dBA lower for the soft ground case due to the different ground type. Otherwise, the BEM plots still show a mitigated ground effect at $x=20 \mathrm{~m}$, which is absent from the hybrid method plots. The BEM plots also show the same source spreading, which again is not modeled in the engineering/hybrid methods.

Again, the hybrid method [plot (d)] does a much better job of approximating the levels of the T-barrier [plot (b)] than the engineering method [plot (c)] does, and Table II quantifies the improvement. Specifically, when the engineering method is naively applied to the Tbarrier using only an I-barrier, the RMS error (1.47 dBA) more than triples compared to the I-barrier (0.41 dBA), but using the hybrid method produces a much smaller RMS error (0.66 dBA).

TABLE II. Error for the soft ground case. Here, $\mu$ is the mean error, $\sigma$ is the standard deviation, and RMS is the root mean squared error. All of the values are in dBA.

\begin{tabular}{lcccc}
\hline \hline Method & Barrier & $\mu$ & $\sigma$ & RMS \\
\hline Harmonoise & $\mathrm{I}$ & -0.02 & 0.41 & 0.41 \\
Harmonoise & $\mathrm{T}$ & 1.31 & 0.67 & 1.47 \\
Hybrid-hard & $\mathrm{T}$ & -0.16 & 0.64 & 0.66 \\
Hybrid-soft & $\mathrm{T}$ & 0.11 & 0.55 & 0.56 \\
\hline \hline
\end{tabular}


To directly test the impact of using a different ground type for the table of corrections, the hybrid method was rerun using a table of corrections that was calculated using soft ground. These results are not shown in Figure 6 because the pictures are very similar but are summarized in the last line of Table II. Including the correct ground type decreases the RMS error from $0.66 \mathrm{dBA}$ to $0.56 \mathrm{dBA}$. In many cases, this $0.1 \mathrm{dBA}$ decrease is likely not worth the extra costs (i.e. having to calculate and store many more tables of corrections). Again, this error is smaller than the error of the standard engineering method compared to BEM for the I-barrier.

Contrasting the results in Tables I and II indicates that the extra attenuation tends to under correct for hard ground but over correct for soft ground. In detail, for hard ground the mean error of the hybrid method for the T-barrier is between the mean errors for the engineering method for the I-barrier and T-barrier (i.e. $-0.28 \mathrm{dBA}<-0.10 \mathrm{dBA}<$ 1.65 dBA), but for soft ground it is less than both of the other mean errors using the engineering method (i.e. $-0.16 \mathrm{dBA}<-0.02 \mathrm{dBA}<1.31 \mathrm{dBA}$ ). More cases must be studied to learn if this result is a trend or is case dependent.

\section{Buildings test case}

The last test case is a T-barrier with buildings and is designed to evaluate the reflection, lateral diffraction, and multiple diffraction approximations of the hybrid method. This case augments the hard ground case, so most of the parameters are the same. The most important change is the two buildings. Figure 7 illustrates the building dimensions and locations, and Table III gives the positions of the corners of the buildings. All of the building surfaces are 
hard, and lateral diffractions are allowed around the buildings. Two additional changes are that the barrier is now only $35 \mathrm{~m}$ long and that the frequency range is only $50 \mathrm{~Hz}-1.6 \mathrm{kHz}$. These changes are necessary to use a fully 3D reference model.

The last important change is the reference method for calculating the reference results (note that the table of corrections is still computed with 2.5D BEM). This case is truly 3D so the reference method must be 3D. However, 3D BEM can only reach up to about $400 \mathrm{~Hz}$ for this scene. Instead, fast-multipole BEM (FM-BEM) $)^{38-40}$ provides the reference levels up to $1.6 \mathrm{kHz}$. FM-BEM is significantly faster than conventional BEM [i.e. $O(n \log n)$ versus $O\left(n^{3}\right)$ ] because it 1) makes the matrix sparse by putting the boundary contributions into a hierarchy, 2) uses an iterative solver, and 3) does not store the entire matrix equation. The FM-BEM implementation was developed at LMSSC/CNAM and was verified using 3D BEM for this geometry up to $400 \mathrm{~Hz}$.

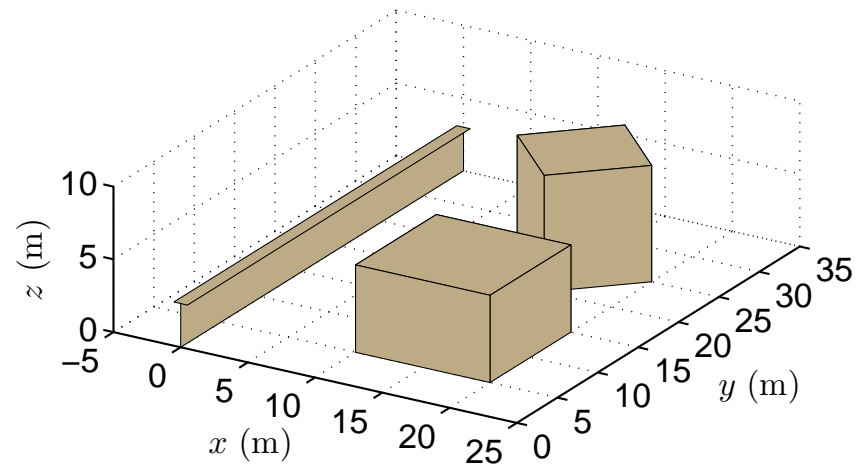

FIG. 7. (Color online) Scene geometry. All surfaces are hard, and the buildings are $6 \mathrm{~m}$ and $8 \mathrm{~m}$ tall.

For the I-barrier (Figure 8), Harmonoise and FM-BEM predict similar levels between the barrier and the buildings but very different levels behind the buildings (i.e. Harmonoise 
TABLE III. Geometry of the buildings. The variables $x, y$, and $z$ are all in meters.

\begin{tabular}{|c|c|c|c|c|c|c|}
\hline & \multicolumn{3}{|c|}{ Building 1} & \multicolumn{3}{|c|}{ Building 2} \\
\hline Corner & $x$ & $y$ & $z$ & $x$ & $y$ & $z$ \\
\hline 1 & 10 & 5 & 6 & 15 & 20 & 8 \\
\hline 2 & 20 & 5 & 6 & 20 & 25 & 8 \\
\hline 3 & 20 & 15 & 6 & 15 & 30 & 8 \\
\hline 4 & 10 & 15 & 6 & 10 & 25 & 8 \\
\hline
\end{tabular}

predicts much lower levels behind the buildings). The larger error in these regions is expected because all of the propagation paths to these receivers are diffracted over or around multiple edges and the engineering methods use simple approximations for multiple diffraction. These differences for the I-barrier also affect the predictions for the T-barrier so that the hybrid method does well between the barrier and the buildings and less so behind the buildings. In practice, the sources are usually incoherent line sources instead of a point source, which decreases the size of this region. Furthermore, the priority is often on accurately predicting the highest levels instead of the lowest levels because they have a greater negative impact on people's well-being.

Since the results are very different in front of and behind the buildings, Table IV provides a statistical summary in front of the buildings (i.e. where $2 \leq x \leq 8$ and $2 \leq y \leq 33$ ) and Table $\mathrm{V}$ provides it behind the buildings (i.e. where $22 \leq x \leq 25$ and $2 \leq y \leq 33$ ). These 

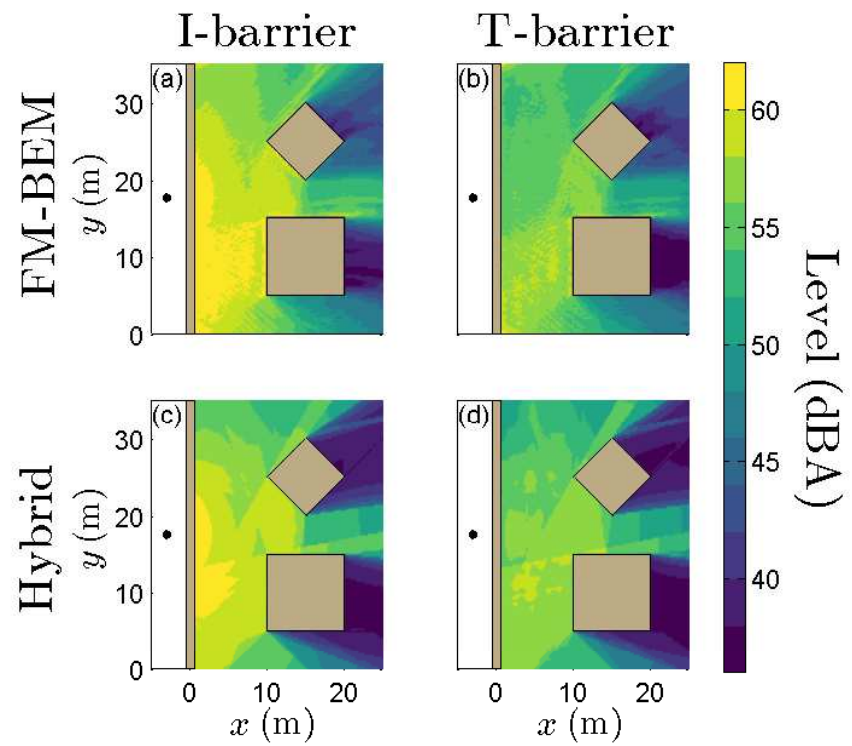

FIG. 8. (Color online) I-barrier (left) or T-barrier (right) with two buildings using FM-BEM (top) or the hybrid method (bottom).

tables demonstrate larger RMS error behind the buildings than in front of them for all of the methods.

TABLE IV. Error for the buildings case in front of the buildings. Here, $\mu$ is the mean error, $\sigma$ is the standard deviation, and RMS is the root mean squared error. All of the values are in dBA.

\begin{tabular}{|c|c|c|c|c|}
\hline Method & Barrier & $\mu$ & $\sigma$ & RMS \\
\hline Harmonoise & I & -0.70 & 0.60 & 0.92 \\
\hline Harmonoise & $\mathrm{T}$ & 2.54 & 1.07 & 2.75 \\
\hline Hybrid & $\mathrm{T}$ & 0.25 & 0.82 & 0.85 \\
\hline
\end{tabular}


TABLE V. Error for the buildings case behind the buildings. Here, $\mu$ is the mean error, $\sigma$ is the standard deviation, and RMS is the root mean squared error. All of the values are in dBA.

\begin{tabular}{|c|c|c|c|c|}
\hline Method & Barrier & $\mu$ & $\sigma$ & RMS \\
\hline Harmonoise & I & -3.2 & 2.5 & 4.1 \\
\hline Harmonoise & $\mathrm{T}$ & -1.5 & 3.0 & 3.3 \\
\hline Hybrid & $\mathrm{T}$ & -2.2 & 2.8 & 3.6 \\
\hline
\end{tabular}

However, Table $\mathrm{V}$ is very different because the mean error of the engineering method is 423 424 tween the I-barrier using Harmonoise (0.92 dBA) and the T-barrier using the hybrid method (0.85 dBA) but much larger for the T-barrier using Harmonoise (2.75 dBA). In addition, the standard deviation increases slightly going from the I-barrier with Harmonoise to the T-barrier with the hybrid method (i.e. from $0.60 \mathrm{dBA}$ to $0.82 \mathrm{dBA}$ ). A small part of this increase is due to the interpolation error and the differences between 2.5D BEM and FM-BEM at the points in the table of corrections. The majority increase in the standard deviation is likely due to the reflection approximation because it ignores the phase information. For comparison, this increase is about the same size as the increase in the standard deviation due to using a table of corrections that is calculated with a different kind of ground (i.e. $0.41 \mathrm{dBA}$ to $0.64 \mathrm{dBA}$ in Table II).

TABLE V. Error for the builings case behind the buildings. Here, $\mu$ is the mean error, $\sigma$ is the

$-3.2 \mathrm{dBA}$. This case demonstrates that the engineering method is less accurate for cases with multiple diffraction. Furthermore, since the T-barrier does not have the largest path length

Table IV shows very similar results to Tables I and II. First, the RMS error is similar be- 
difference, the error in the engineering method is larger than the T-top correction. This observation justifies the modeling choice of not calculating a correction when the complex object does not have the largest path length difference. Moreover, the RMS error is actually lower modeling the T-barrier with Harmonoise instead of the hybrid method because 1) the mean error for the I-barrier is negative, 2) the extra attenuation is also negative, and 3) the absolute value of the mean error for the I-barrier is larger than the absolute value of the mean extra attenuation. In contrast, one trend that continues from the previous tables is that the standard deviation is smallest for the I-barrier, slightly larger for the T-barrier using the hybrid method, and the largest for the T-barrier using Harmonoise. This result suggest that the hybrid method would do better than Harmonoise for the T-barrier if Harmonoise modeled the I-barrier more accurately. Nonetheless, these multiple diffraction or lateral diffraction paths are not usually the largest contributor to the level when the source is one or more incoherent line sources.

\section{CONCLUSION}

This paper develops and validates a hybrid approach to model complex geometries in large urban settings by combining a standard noise engineering method with 2.5D BEM. BEM provides a table of corrections that quantifies the impact of a complex object compared to a simplified object in a very simple surrounding (i.e. usually hard flat ground). For each propagation path and as required, the engineering method linearly interpolates this dataset based on the geometry of the propagation path to obtain the extra attenuation associated with the complex object. 
The hybrid method is used to model a T-barrier with hard ground, soft ground, and buildings. All of these cases demonstrate that the hybrid method has better accuracy than the standard engineering methods and is far more computationally efficient than full 3D reference methods. Indeed, the hybrid method is computationally efficient enough to be applied whenever the standard engineering methods can be used. The domain size of the validation cases is not limited by the capabilities of the hybrid method but rather of the reference method.

Since the hybrid method has many potential sources of error, it has many opportunities for improvement. First, the predictions of the standard engineering methods do not perfectly match the predictions of reference methods, and any improvement to the engineering methods would also improve the hybrid method. Second, the hybrid method also has some moderate interpolation error. Since the validation section only used 396 source/receiver points, the best way to decrease the interpolation error is to increase the number of points in the table of corrections. However, the total number of points is limited by the available computer memory, so eventually the positions could also be optimized to reduce the interpolation error. Third, many of the approximations (e.g. the multiple and lateral diffraction) are very simple because there are larger errors elsewhere. If the error of the underline engineering method is decreased, then these approximations should be revised.

\section{ACKNOWLEDGMENTS}

This research was supported by the Centre Scientifique et Technique du Bâtiment (CSTB). 


\section{REFERENCES}

${ }^{1}$ L. Fritschi, A. L. Brown, R. Kim, D. Schwela, and S. Kephalopoulos, "Burden of disease from envirnmental noise: quantification of healthy life years lost in Europe," World Health Organization Regional Office for Europe (2011), www.euro.who.int/ en/publications/abstracts/burden-of-disease-from-environmental-noise.

-quantification-of-healthy-life-years-lost-in-europe.

2 "Directive 2002/49/EC of the European parliament and the council of 25 June 2002 relating to the assessment and meanagement of envirnmental noise," (2002), eur-lex.europa. eu/legal-content/EN/TXT/?uri=CELEX : 32002L0049.

${ }^{3}$ S. Kephalopoulos, M. Paviotti, and F. Anfosso-Lédée, "Common noise assessment methods in Europe (CNOSSOS-EU)," European Commission Joint Research Centre (JRC) (2012), doi: $10.2788 / 31776$.

${ }^{4}$ L. L. Thompson, "A review of finite-element methods for time-harmonic acoustics," J. Acoust. Soc. Am. 119(3), 1315-1330 (2006) doi: 10.1121/1.2164987.

${ }^{5}$ I. Harari, "A survey of finite element methods for time-harmonic acoustics," Comput. Methods Appl. Mech. Eng. 195(13-16), 1594-1607 (2006) doi: 10.1016/j .cma.2005.05. 030.

${ }^{6}$ D. C. Hothersall, S. N. Chandler-Wilde, and M. N. Hajmirzae, "Efficiency of single noise barriers," J. Sound Vib. 146(2), 303-322 (1991) doi: 10.1016/0022-460X (91)90765-C.

${ }^{7}$ M. Baulac, J. Defrance, P. Jean, and F. Minard, "Efficiency of noise protections in urban areas: predictions and scale model measurements," Acta Acust. united with 
Acust. 92(4), 530-539 (2006) www.ingentaconnect.com/contentone/dav/aaua/2006/ 00000092/00000004/art00004.

${ }^{8} \mathrm{~T}$. Van Renterghem, "Efficient outdoor sound propagation modeling with the finitedifference time-domain (FDTD) method: a review," Int. J. Aeroacoustics 13(5-6), 385-404 (2014) doi: $10.1260 / 1475-472 x \cdot 13 \cdot 5-6.385$.

${ }^{9}$ R. Mehra, N. Raghuvanshi, A. Chandak, D. G. Albert, D. K. Wilson, and D. Manocha, "Acoustic pulse propagation in an urban environment using a three-dimensional numerical simulation," J. Acoust. Soc. Am. 135(6), 3231-3242 (2014) doi: 10.1121/1.4874495.

${ }^{10} \mathrm{P}$. Jean, "A variational approach for the study of outdoor sound propagation and application to railway noise," J. Sound Vib. 212(2), 275-294 (1998) doi: 10.1006/jsvi.1997. 1407.

${ }^{11}$ T. Ishizuka and K. Fujiwara, "Performance of noise barriers with various edge shapes and acoustical conditions," Appl. Acoust. 65(2), 125-141 (2004) doi: 10.1016/j .apacoust. 2003.08 .006$.

${ }^{12}$ M. Baulac, J. Defrance, and P. Jean, "Optimisation with genetic algorithm of the acoustic performance of T-shaped noise barriers with a reactive top surface," Appl. Acoust. 69(4), 332-342 (2008) doi: 10.1016/j.apacoust.2006.11.002.

${ }^{13}$ A. Jolibois, D. Duhamel, V. W. Sparrow, J. Defrance, and P. Jean, "Sensitivity-based shape optimization of a rigid tramway low-height noise barrier," in Inter-noise Proc., Innsbruck, Austria (2013), pp. 3492-3501, www.ingentaconnect.com/contentone/ince/ incecp/2013/00000247/00000004/art00013. 
Outdoor noise propagation with complex geometries

14 "ISO 9613-2:1996 Acoustics - attenuation of sound during propagation outdoors - part 2: general method of calculation," International Organization for Standardization (ISO) (1996), www.iso.org/standard/20649.html.

${ }^{15}$ D. van Maercke and J. Defrance, "Development of an analytical model for outdoor sound propagation within the Harmonoise project," Acta Acust. united with Acust. 93(2), 201-212 (2007) www.ingentaconnect.com/contentone/dav/aaua/2007/ 00000093/00000002/art00004.

${ }^{16}$ E. Salomons, D. van Maercke, J. Defrance, and F. de Roo, "The Harmonoise sound propagation model," Acta Acust. united with Acust. 97(1), 62-74 (2011) doi: 10.3813/AAA. 918387.

${ }^{17}$ D. P. Hewett, "High frequency sound propagation in a network of interconnecting streets," J. Sound Vib. 331(25), 5537-5561 (2012) doi: 10.1016/j.j.sv.2012.07.030.

${ }^{18}$ A. Muradali and K. R. Fyfe, "Accurate barrier modeling in the presence of atmospheric effects," Appl. Acoust. 56(3), 157-182 (1999) doi: 10.1016/S0003-682X (98)00023-1.

${ }^{19}$ A. Farina, "Validation of the pyramid tracing algorithm for sound propagation outdoors: comparison with experimental measurements and with the ISO-DIS 9613 standards," Adv. Eng. Softw. 31(4), 241-250 (2000) doi: 10.1016/S0965-9978(99)00053-8.

${ }^{20}$ H. Wang, Z. Yu, and M. Cai, "The 3D attenuation calculation of traffic noise among building groups by using beam tracing method," Procedia - Soc. Behav. Sci. 96, 19291937 (2013) doi: 10.1016/j.sbspro.2013.08.218. 
${ }^{21}$ D. Lee, A. D. Pierce, and E. Shang, "Parabolic equation development in the twentieth century," J. Comput. Acoust. 8(4), 527-637 (2000) doi: 10.1142/S0218396X00000388.

${ }^{22}$ T. Van Renterghem, D. Botteldooren, and P. Lercher, "Comparison of measurements and predictions of sound propagation in a valley-slope configuration in an inhomogeneous atmosphere," J. Acoust. Soc. Am. 121(5), 2522-2533 (2007) doi: 10.1121/1.2717765.

${ }^{23}$ F. Aballéa and J. Defrance, "Single and multiple reflections in plane obstacle using the parabolic equation method with a complementary Kirchhoff approximation," Acta Acust. united with Acust. 93(1), 22-30 (2007) www.ingentaconnect.com/contentone/ dav/aaua/2007/00000093/00000001/art00003.

${ }^{24}$ T. Van Renterghem, E. M. Salomons, and D. Botteldooren, "Efficient FDTD-PE Model for sound propagation in situations with complex obstacles and wind profiles," Acta Acust. united with Acust. 91(4), 671-679 (2005) www.ingentaconnect.com/contentone/dav/ aaua/2005/00000091/00000004/art00006.

${ }^{25}$ J. Defrance, E. Salomons, I. Noordhoek, D. Heimann, B. Plovsing, G. Watts, H. Jonasson, X. Zhang, E. Premat, I. Schmich, F. Aballea, M. Baulac, and F. de Roo, "Outdoor sound propagation reference model developed in the European Harmonoise project," Acta Acust. united with Acust. 93(2), 213-227 (2007) www.ingentaconnect.com/contentone/dav/ aaua/2007/00000093/00000002/art00005.

${ }^{26}$ P. Jean, "Coupling integral and geometrical representations for vibro-acoustical problems," J. Sound Vib. 224(3), 475-487 (1999) doi: 10.1006/jsvi.1999.2195. 
Outdoor noise propagation with complex geometries

${ }^{27} \mathrm{P}$. Jean, "Coupling geometrical and integral methods for indoor and outdoor sound propagation - validation examples," Acta Acust. united with Acust. 87(2), 236-246 (2001) www. ingentaconnect.com/contentone/dav/aaua/2001/00000087/00000002/art00010.

${ }^{28}$ S. Hampel, S. Langer, and A. Cisilino, "Coupling boundary elements to a raytracing procedure," Int. J. Numer. Methods Eng. 73(3), 427-445 (2008) doi: 10.1002/nme. 2080.

${ }^{29}$ H. Yeh, R. Mehra, Z. Ren, L. Antani, D. Manocha, and M. Lin, "Wave-ray coupling for interactive sound propagation in large complex scenes," ACM Trans. Graph. 32(6), 165:1-11 (2013) doi: 10.1145/2508363.2508420.

${ }^{30} \mathrm{~J}$. Defrance and P. Jean, "Integration of the efficiency of noise barrier caps in a 3D ray tracing method. case of a T-shaped diffracting device," Appl. Acoust. 64(8), 765-780 (2003) doi: 10.1016/S0003-682X(03)00034-3.

${ }^{31}$ C. H. Kasess, W. Kreuzer, and H. Waubke, "Deriving correction functions to model the efficiency of noise barriers with complex shapes using boundary element simulations," Appl. Acoust. 102, 88-99 (2016) doi: 10.1016/j.apacoust.2015.09.009.

${ }^{32}$ B. Plovsing, "Nord2000. Comprehensive outdoor sound propagation model. part 1: propagation in an atmosphere without significant refraction," Danish Electronics, Light, and Acoustics (DELTA) (2006), dk.madebydelta.com/viden/publikationer/.

${ }^{33}$ D. van Maercke, T. Leissing, and J. Maillard, "Task 6.2: Evaluation of innovative and combined mitigations," Holistic and sustainable abatement of noise by optimized combinations of natural and artificial means (HOSANNA) (2013), www.greener-cities.eu/. 
${ }^{34}$ D. Duhamel, "Efficient calculation of the three-dimensional sound pressure field around a noise barrier," J. Sound Vib. 197(5), 547-571 (1996) http://www.sciencedirect.com/ science/article/pii/S0022460X96905489 doi: 10.1006/jsvi.1996.0548.

${ }^{35}$ P. Jean, J. Defrance, and Y. Gabillet, "The importance of source type on the assessment of noise barriers," J. Sound Vib. 226(2), 201-216 (1999) http://www.sciencedirect. com/science/article/pii/S0022460X99922733 doi: 10.1006/jsvi.1999.2273.

${ }^{36}$ J. Defrance, P. Jean, F. Koussa, A. Khan, K. Horoshenkov, H. Benkreira, T. Van Renterghem, J. Kang, J. Smyrnowa, and J. Forssén, "Task 2.3: Application to innovations," Holistic and sustainable abatement of noise by optimized combinations of natural and artificial means (HOSANNA) (2013), www.greener-cities.eu/.

${ }^{37}$ M. Delany and E. Bazley, "Acoustical properties of fibrous absorbent materials," Appl. Acoust. 3(2), 105-116 (1970) doi: 10.1016/0003-682X (70)90031-9.

${ }^{38}$ T. Sakuma and Y. Yasuda, "Fast multipole boundary element nethod for large-scale steady-state sound field analysis. part I : setup and validation," Acta Acust. united with Acust. 88(4), 513-525 (2002) www.ingentaconnect.com/contentone/dav/aaua/2002/ 00000088/00000004/art00007.

${ }^{39} \mathrm{X}$. Vuylsteke, "Development of a reference method based on the fast multipole boundary element method for sound propagation problems in urban environments: formalism, optimizations \& applications," Doctoral dissertation, Université Paris-Est, 2014, tel.archives-ouvertes.fr/tel-01167130/document. 
Outdoor noise propagation with complex geometries

589

590

591

${ }^{40}$ N. A. Gumerov and R. Duraiswami, Fast multipole methods for the Helmholtz equation in three dimensions (Elsevier, 2004), pp. 1-520, www.sciencedirect.com/science/book/ 9780080443713. 